\title{
The Effects of Microdecompression on Patients with Lumbar Degenerative Spinal Stenosis with or without Degenerative Spondylolisthesis
}

\author{
Mustafa OGDEN ${ }^{1}$, Ulas YUKSEL ${ }^{2}$, Bulent BAKAR ${ }^{1}$, Suleyman AKKAYA ${ }^{1}$, Kagan KAMASAK ${ }^{3}$, Ahmet Turan DAGLI ${ }^{3}$ \\ ${ }^{1}$ Kirikkale University, Faculty of Medicine, Department of Neurosurgery, Kirikkale, Turkey \\ ${ }^{2}$ Sanliurfa Training and Research Hospital, Neurosurgery Clinic, Sanliurfa, Turkey \\ ${ }^{3}$ Hitit University, Faculty of Medicine, Department of Neurosurgery, Corum, Turkey
}

This study has been presented as an oral presentation at the $16^{\text {th }}$ WFNS World Congress of Neurosurgery between 20 and 25 August 2017 at Istanbul, Turkey.

Corresponding author: Mustafa OGDEN mustafaogden38@gmail.com

\section{ABSTRACT}

AIM: To investigate the mid-term efficacy of bilateral decompression with a unilateral approach (BDUA) on symptoms and quality of life of in patients with lumbar spinal stenosis (LSS), with low grade (with percentage slip <25\%) degenerative spondylolisthesis or without spondylolisthesis.

MATERIAL and METHODS: The study included patients who underwent BDUA due to one or two-level LSS related to degenerative spondylosis and/ or degenerative spondylolisthesis. Pre- and postoperative data of the Oswestry Disability Index (ODI), Visual Analogue Scale (VAS), and walking distance were compared.

RESULTS: Evaluation was made up of a total of 53 patients, comprising 32 males and 21 females with a mean age of $60.6 \pm$ 9.0 years. Compared with the preoperative data, VAS, ODI and walking distance improved significantly in all patients at the 3-year follow-up examination. Furthermore, a significant improvement was determined in the clinical parameters of patients with degenerative spondylolisthesis, with no worsening of slip and no requirement for instrumentation. Moreover, when patients were separated into two groups according to age, as elderly ( $>60$ years) and adult ( $<60$ years), similar improvements were seen in both groups.

CONCLUSION: BDUA could be quite effective in reducing pain, improving quality of life and walking distance with no worsening of the grade or degree of slipping.

KEYWORDS: Lumbar, Stenosis, Spondylolisthesis, Microsurgery, Hemilaminectomy

\section{INTRODUCTION}

$\mathrm{L}$ umbar spinal stenosis (LSS), may have an unfavorable impact on activities of daily living and walking distance lbecause of pain in the lower limbs when walking (5). Degenerative stenosis is a condition that occurs as a result of age-related degeneration in the vertebrae. The problem here is not only the degeneration of the facet joints, but also ligamentum flavum hypertrophy and central degenerative disc herniation. This is a clinical and radiological condition seen in more than $65 \%$ of females and $80 \%$ of males, with incidence directly related to age. In addition, degenerative spondylolisthesis, basically a facet joint lesion, develops as a consequence of degeneration of the lumbar vertebrae, resulting in hypertrophy and segmental instability in the facet joints. With age, the inferior and superior articular processes of the
Mustafa OGDEN (1) : 0000-0002-7129-0936 Ulas YUKSEL (1) : 0000-0002-6398-4110
Bulent BAKAR (1): 0000-0002-6236-7647 Suleyman AKKAYA (D): 0000-0003-0597-1861
Kagan KAMASAK (D) : 0000-0002-0404-9667 Ahmet Turan DAGLI (D) : 0000-0002-9214-8138 
facet joint are shifted due to remodeling. Slip in degenerative spondylolisthesis does not exceed $30 \%$ of the vertebral body and degenerative spondylolisthesis does not contain a pars interarticularis defect (7). Machado et al. reviewed different surgical methods for decompression and widening the spinal canal in LSS and reported that decompression plus fusion, or expanding the interspinous process spaces was not superior to conventional decompression alone $(12,17)$. Many current articles have suggested the avoidance of bilateral laminectomy and instrumentation because bilateral decompression with a unilateral approach (BDUA) has been shown to be effective in reducing pain, increasing walking distance, and improving disability $(1,13,14,16,19,22)$. However, some reports have demonstrated that although unilateral laminectomy is effective in the early postoperative period, it has poor outcomes in the long term (approximately 5 years) $(6,10)$. Moreover, Müslüman et al. recommended that unilateral decompression is not performed on patients with a previous history of lumbar surgery (14).

This study was conducted to discuss these conflicting findings in literature and to investigate the mid-term efficacy of BDUA on symptoms and quality of life of patients, especially in elderly and/ or female patients with low grade (with percentage slip <25\%) degenerative spondylolisthesis or without spondylolisthesis.

\section{MATERIAL and METHODS}

\section{Participants}

All procedures performed in studies involving human participants were in accordance with the ethical standards of the institutional and/or national research committee and with the 1964 Helsinki declaration and its later amendments or comparable ethical standards. For this type of study formal consent is not required. Informed consent was obtained from all individual participants included in the study and no identifying information of any participant is included in this article.

Patient data were obtained retrospectively from the hospital records of patients applied with BDUA. The inclusion and exclusion criteria for the patients are shown below:

\section{Inclusion criteria for the patients}

- One or two adjacent stenotic segments

- Duration of symptoms $>6$ months

- No significant slipping or slipping related to low grade (with percentage slip <25\%) degenerative spondylolisthesis

- History of no response to conventional treatment modalities

\section{Exclusion criteria for the patients}

- LSS due to spondylolysis, tumor or inflammatory changes

- Degenerative lumbar scoliosis

- History of lumbar spinal surgery for spinal stenosis or instability

\section{- Slipping related to the pars interarticularis defect}

- Severe comorbid diseases such as cardiac or lung disease, psychiatric disorders

The age, gender, and pre- and postoperative walking distances (WD) measured in meters were obtained from patient statements. The patient data obtained from the hospital records included comorbid disease (diabetes mellitus, respiratory diseases), duration of the surgical intervention measured in minutes from the time of the first skin incision to the time of skin closure (called TIME), the amount of bleeding during surgery measured in millilitres that was calculated by subtracting the amount of fluid used for irrigation from the total fluid in the aspirate (called BLEEDING) and hospitalization time measured in days (called DISCHARGE).

Lumbar magnetic resonance (MR) images were evaluated preoperatively to diagnose the lumbar spinal stenosis in each patient. To check for any vertebral slipping, preoperative and postoperative ( 3 years after surgery) lumbar X-ray images were obtained with the patient standing in hyperflexion and hyperextension postures.

Preoperatively and at 3 years postoperatively, the levels of pain and disability were determined using the scales described below:

- Oswestry Disability Index (ODI), which has been proven valid and reliable for the Turkish population, was used to evaluate patient disability due to pain or neurological deficits. It consists of 10 items of "severity of pain", "personal care", "rising", "walking", "sitting", "standing", "social life", "sleep", "travelling" and "degree of pain". Each item is scored from 0 to 5 . Higher total points indicate a higher disability level of the patient $(8,21)$.

- Visual Analogue Scale (VAS) was used to evaluate the severity of the buttock/ lower limb pain of the patient, with the lowest point of 0 as no pain and the highest points of 10 as intolerable pain (3).

All evaluations were performed by the same physician.

\section{Surgical Technique}

All patients underwent BDUA under general anesthesia. While the patients were in the prone position, the level was determined by using fluoroscopy. Following the required surgical site preparation, a midline $2 \mathrm{~cm}$ skin incision was made. In cases with radicular pain, paravertebral muscle dissection was performed from the site of the pain. Otherwise the paravertebral muscle dissection was administered from the left side of the patient to be able to use the surgical hand instruments (high-speed drill, fine Kerrison rongeurs, etc) and surgical microscope optimally as the surgeon was right-handed (M.O). If there was a deviated spinous process, it was drilled before reaching the contralateral surgical site. Then, the lamina was elevated sub-periostally and a surgical microscopic view of the area was provided. Before hemilaminectomy and foraminotomy were performed, the lamina was drilled. After rotating the operating table to the contralateral side, the lamina of the opposite side was drilled 
under the spinous process and the flavum was totally removed. Neural foraminas were released and roots were decompressed bilaterally (Figure 1). The intervertebral disc space was checked and microdiscectomy was performed if necessary, except in patients with degenerative spondylolisthesis. After hemostasis was obtained, the anatomic structures were closed appropriately. All the operations were performed by the same neurosurgeon (M.O.).

\section{Statistical Analysis}

After checking the normal distribution of data, the Mann Whitney $U$ test was used for binary comparisons. The Spearman correlation test was used for correlation analyses. Preoperative and postoperative continuous variables were analyzed using the Wilcoxon Signed Rank test. A value of $p$ $<0.05$ was accepted as statistically significant.

\section{RESULTS}

Evaluation was made up of a total of 53 patients, comprising 32 males and 21 females with a mean age of $60.6 \pm 9$ years. All the demographic data are presented in Table I. No significant neurological deficit was observed in any patient.

Postoperative VAS scores, ODI scores and walking distance values were different between the groups when the participants were separated into two groups according to the patients with low grade (percentage slip <25\%) degenerative spondylolisthesis ( $\mathrm{n}: 19,11$ males, 8 females) and patients without spondylolisthesis (n:34). No difference was found between the groups in respect of age, gender, BMI, preoperative VAS scores, preoperative ODI scores, preoperative walking distance values, duration of the surgical intervention, the amount of bleeding during surgery, number
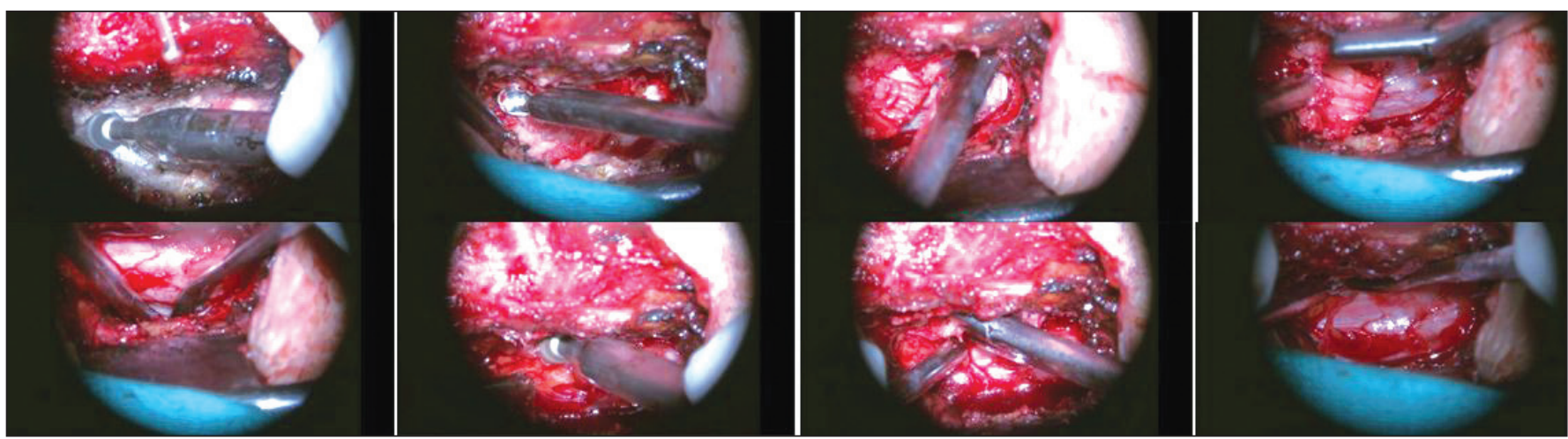

Figure 1: Photographs obtained intraoperatively showing the application of bilateral decompression via unilateral approach (BDUA).

Table I: Descriptive Table Showing the Findings of the Patients Who were Separated into Two Groups according to the Patients with Degenerative Stenosis and the Patients with Degenerative Stenosis and Low Grade Spondylolisthesis

\begin{tabular}{lccc}
\hline Variable & $\begin{array}{c}\text { Patients with degenerative } \\
\text { stenosis (n=34) }\end{array}$ & $\begin{array}{c}\text { Patients with degenerative stenosis and } \\
\text { spondylolisthesis (n=19) }\end{array}$ & p \\
\hline Age (year) & $58 \pm 8.09$ & $61 \pm 10.43$ & 0.348 \\
\hline BMI & $23 \pm 2.64$ & $24 \pm 2.18$ & 0.621 \\
\hline Preop VAS & $9 \pm 1.20$ & $8 \pm 1.73$ & 0.540 \\
\hline Postop VAS & $1 \pm 0.88$ & $3 \pm 1.86$ & 0.008 \\
\hline Preop ODI & $60 \pm 16.70$ & $67 \pm 14.73$ & 0.602 \\
\hline Postop ODI & $18 \pm 2.78$ & $23 \pm 8.28$ & $\mathbf{0 . 0 0 4}$ \\
\hline Preop WD (meter) & $50 \pm 94.20$ & $100 \pm 74.20$ & 0.167 \\
\hline Postop WD (meter) & $2000 \pm 743.68$ & $1000 \pm 800.17$ & $\mathbf{0 . 0 1 4}$ \\
\hline TIME (minute) & $45 \pm 10.67$ & $45 \pm 12.87$ & 0.490 \\
\hline BLEEDING (milliliter) & $50 \pm 27.32$ & $50 \pm 23.86$ & 0.551 \\
\hline DISCHARGE (day) & $1 \pm 4.62$ & $1 \pm 0.01$ & 0.082 \\
\hline
\end{tabular}

BMI: Body mass index, PreopVAS: Preoperative Visual Analogue Scale score, Postop VAS: Postoperative Visual Analogue Scale score, Preop ODI: Preoperative Oswestry Disability Index score, Postop ODI: Postoperative Oswestry Disability Index score, Preop WD: Preoperative walking distance measured in meters, Postop WD: Postoperative walking distance measured in meters, TIME: Duration of the surgery measured in minutes, BLEEDING: Amount of the bleeding during surgery measured in milliliters, DISCHARGE: Duration of the hospitalization time measured in days, $\boldsymbol{n}$ : Number of the patients. 
of decompression levels, hospitalization time and discectomy requirement. Although the postoperative VAS scores and ODI scores were higher and walking distance was lower in patients with degenerative spondylolisthesis, this technique was observed to lead to improvements in the postoperative VAS score, ODI score and walking distance compared to the preoperative values in these patients (Table I).

When the participants were divided into two groups according to age, as the elderly group ( $>60$ years) and the adult group ( $<60$ years), gender (female and male) or the number of the decompressed lumbar levels (one level or two levels), no difference was determined between the groups in respect of the variables $(p>0.05)$. When the participants were divided into two groups according to the surgical intervention as with and without discectomy, no difference was found between the groups in respect of the variables ( $p>0.05)$. In the evaluation according to the number of decompressed lumbar levels, age and gender, it was observed that the postoperative VAS score, ODI score and walking distance improved significantly compared to the preoperative findings $(p<0.001)$. No change was determined between the pre- and postoperative grade of vertebral slipping on the radiological images of the patients ( $p>0.05$ ) (Figures 2A, B; 3A, B; 4A, B; 5A, B).

\section{Correlation Analysis}

In all patients, there was a positive correlation between age and operation time, between the preoperative VAS and preoperative ODI scores. The postoperative VAS score was positively correlated to the pre- and postoperative slipping scores and postoperative ODI scores. The postoperative ODI scores were positively correlated to the pre- and postoperative slipping scores. A positive correlation was determined between the pre- and postoperative walking distance values.
A negative correlation was found between the postoperative walking distance values and the pre- and postoperative vertebral slipping scores. Hospitalization time was positively correlated with the absence of comorbid diseases such as diabetes mellitus and respiratory diseases. No correlation was found between the preoperative vertebral slipping and postoperative vertebral slipping scores, between BMI and pre- and postoperative slipping scores, between BDUA with discectomy and pre- and postoperative vertebral slipping scores.

In the adult patient group, there was a positive correlation between age and BMI, between preoperative VAS scores and preoperative ODI scores, between postoperative VAS scores and pre- and postoperative slipping scores, between preoperative and postoperative walking distance values, between the duration of the surgical intervention and BMI, between hospitalization time and BDUA with discectomy. There was a negative correlation between the postoperative VAS score and the amount of bleeding during surgery.

In the elderly patient group, there was a positive correlation between age and postoperative VAS scores, between postoperative VAS and postoperative ODI scores, between postoperative ODI score and pre- and postoperative slipping scores, between postoperative VAS scores and the amount of bleeding during surgery. A negative correlation was found between age and preoperative VAS scores, between age and postoperative walking distance value, between preoperative VAS score and postoperative walking distance value, between preoperative walking distance and pre- and postoperative vertebral slipping scores between preoperative ODI score and hospitalization time, and between the duration of the surgical intervention and BMI values.
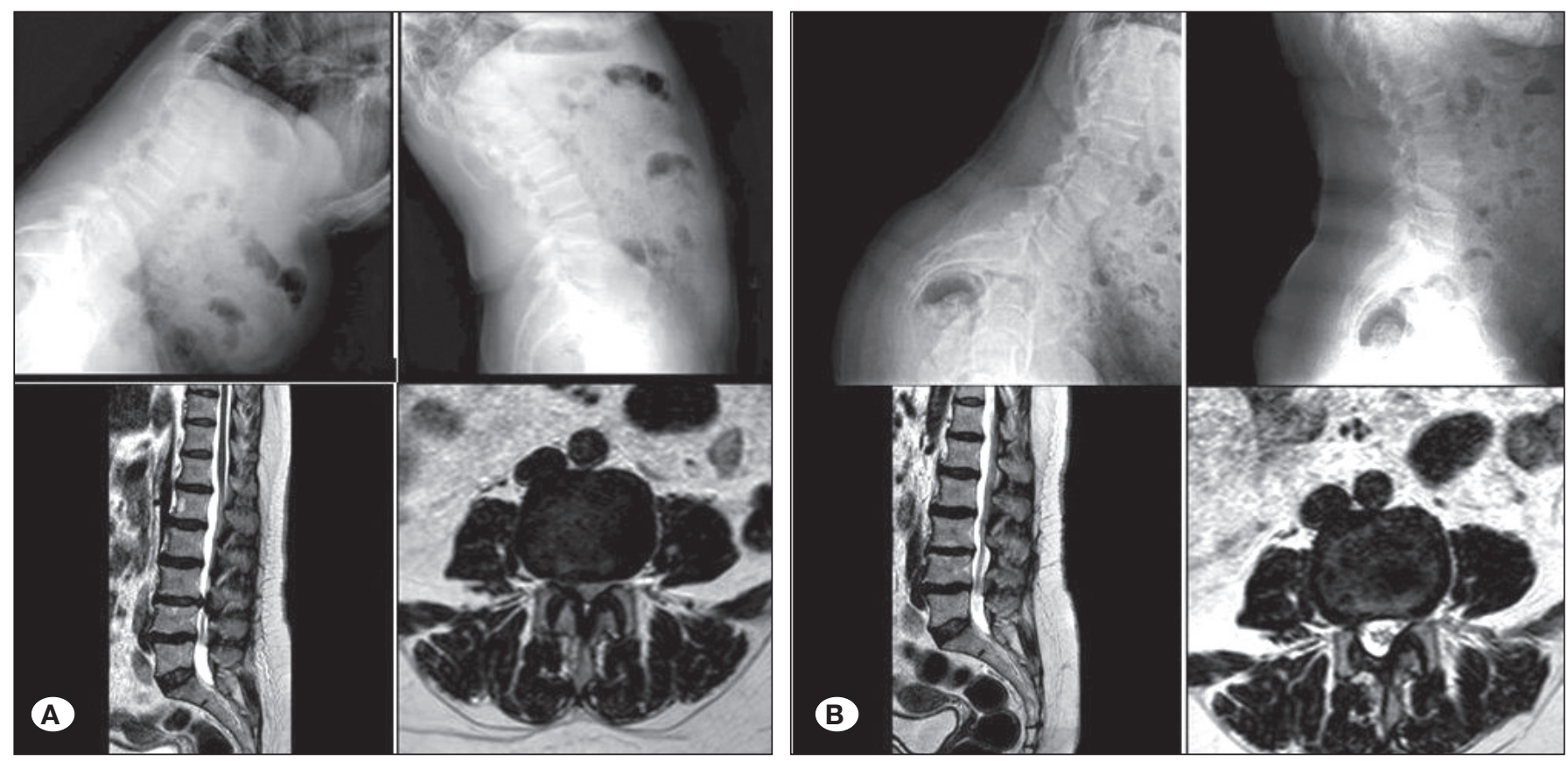

Figure 2: Radiological images of a patient with one-level lumbar spinal stenosis without vertebral slipping (A); no slipping occurred after the surgical intervention (B). 

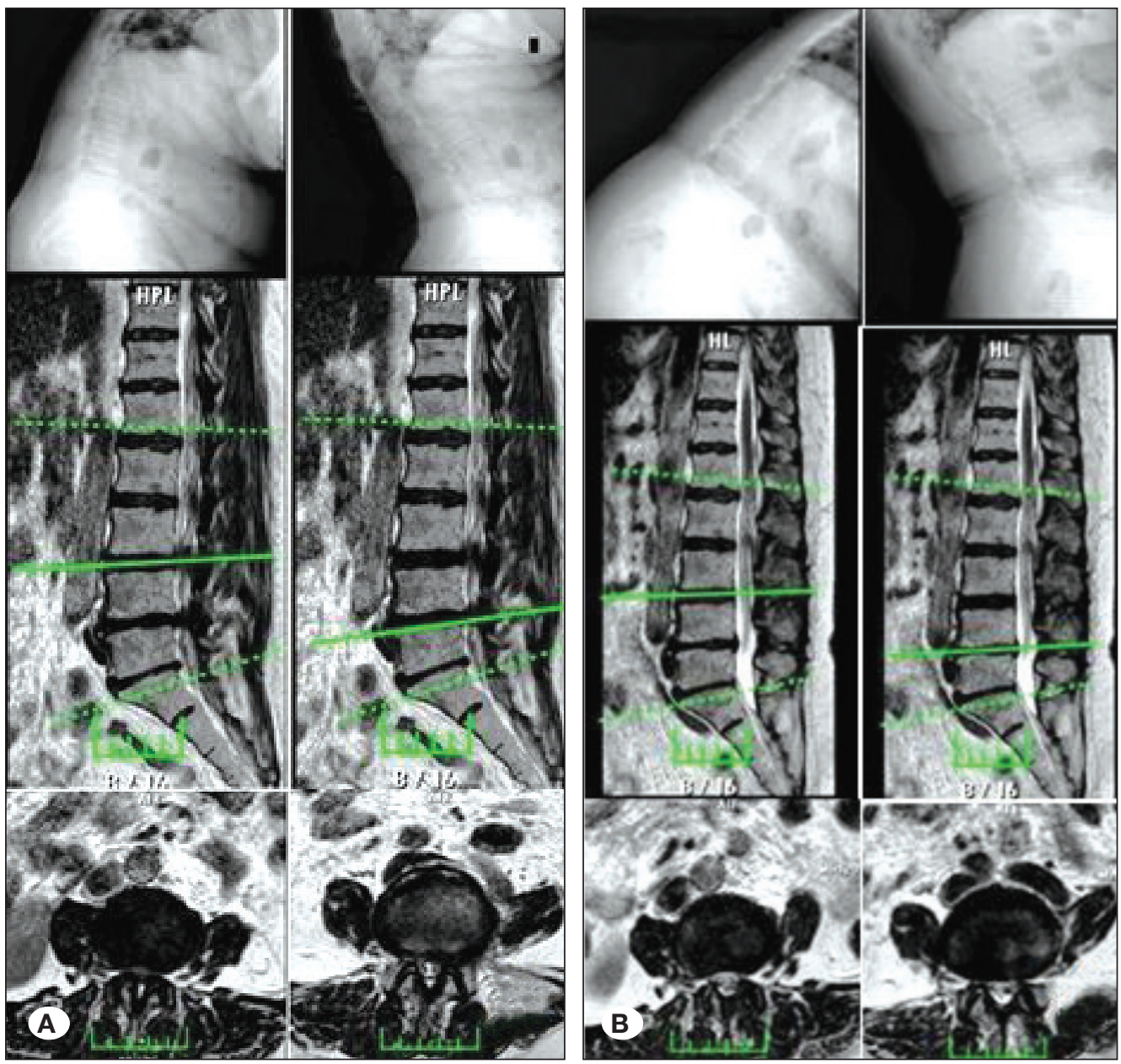

Figure 3: Radiological images of a patient with two-level lumbar spinal stenosis without vertebral slipping (A); no vertebral slipping occurred postoperatively (B).
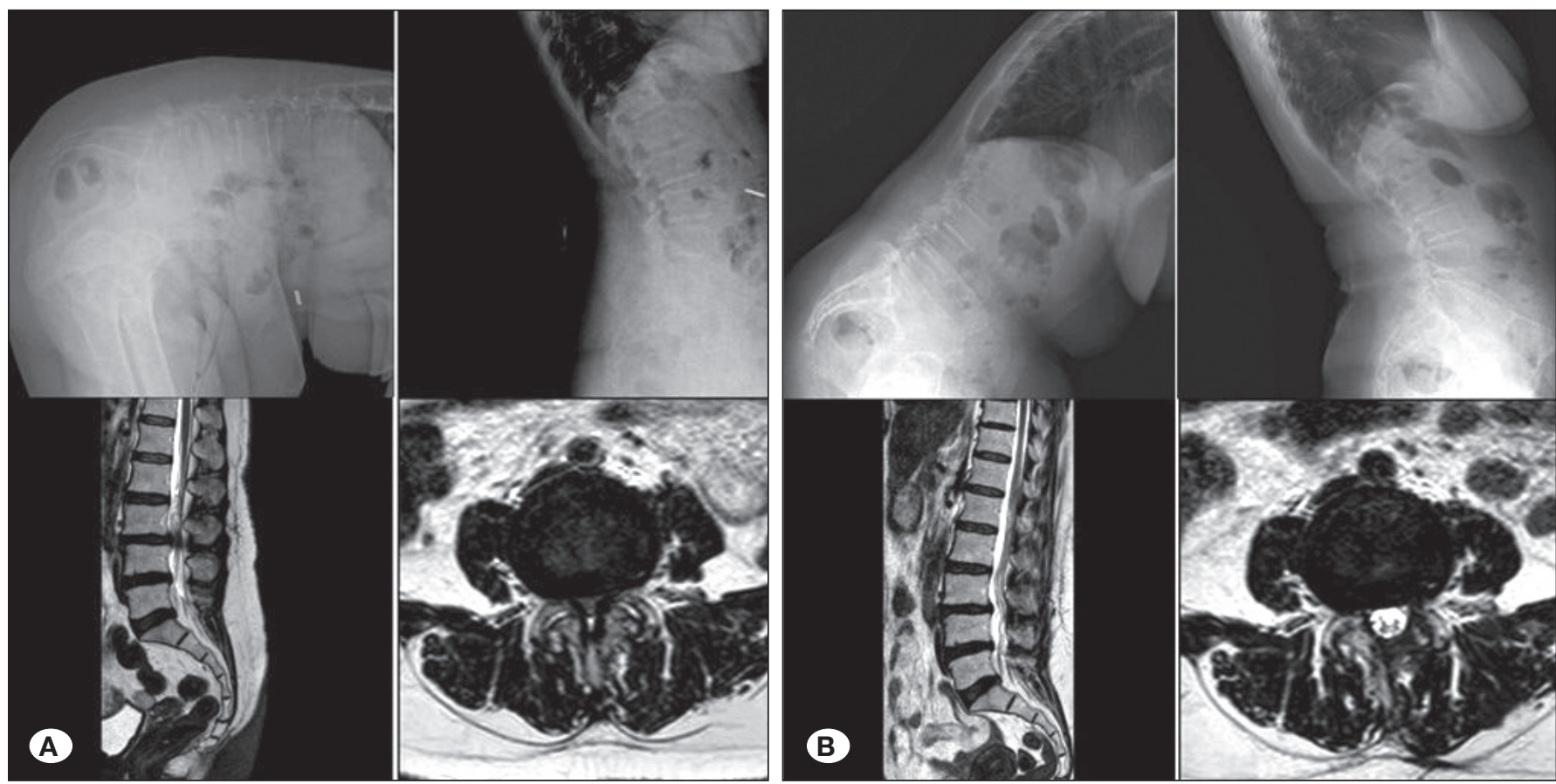

Figure 4: Radiological images of a patient with one-level lumbar spinal stenosis related to vertebral slipping (A); surgery did not increase the vertebral slipping grade postoperatively (B). 

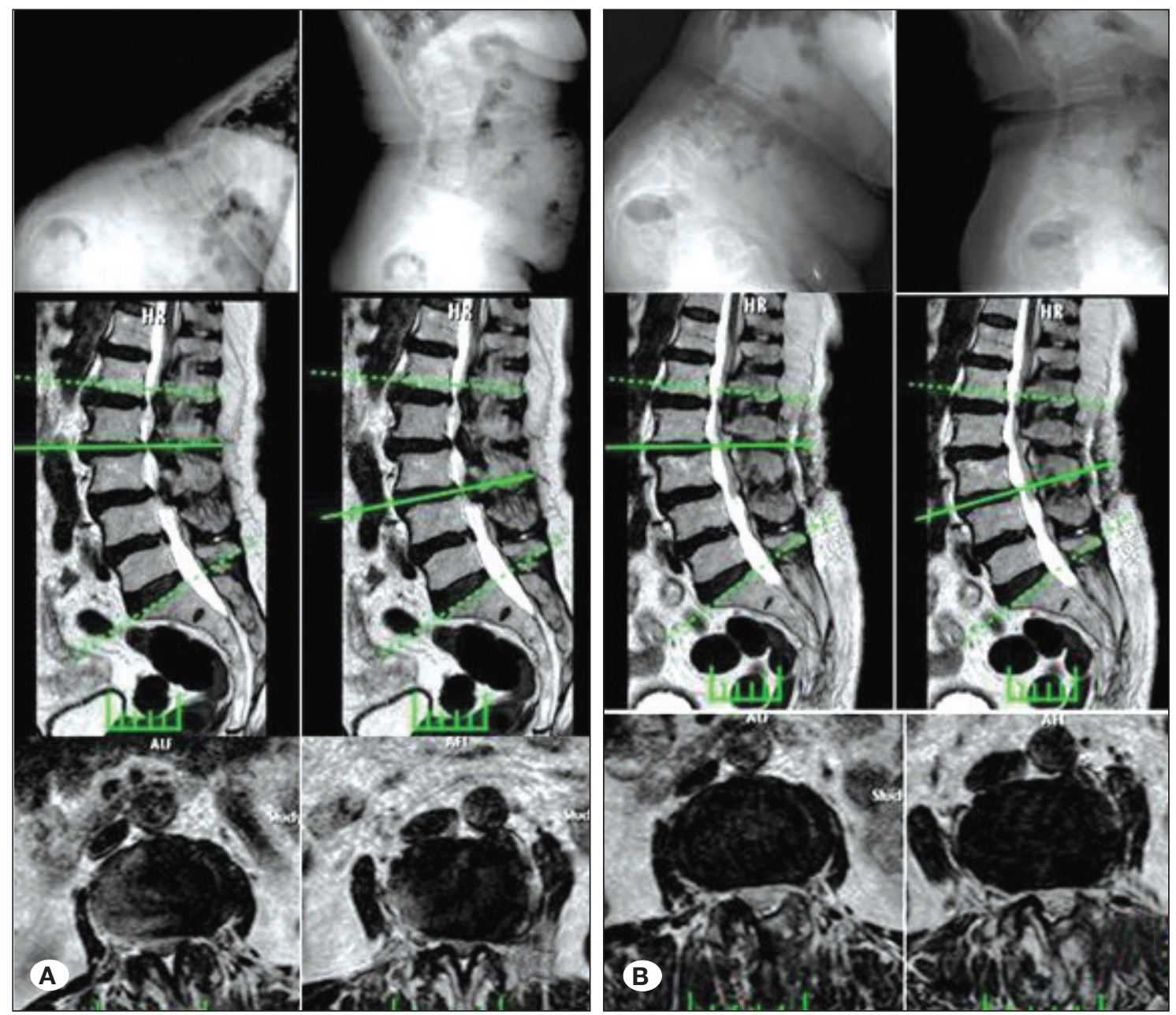

Figure 5:

Radiological images of a patient with two-level lumbar spinal stenosis related to vertebral slipping (A); surgery did not increase the vertebral slipping grade postoperatively (B).
In female patients, there was a positive correlation between preoperative VAS and preoperative ODI scores, and between postoperative VAS score and the pre- and postoperative vertebral slipping score. In male patients, there was a positive correlation between age and the duration of the surgical intervention, between postoperative VAS and postoperative ODI scores, between postoperative ODI score and pre- and postoperative vertebral slipping scores. A negative correlation was found between preoperative VAS scores and the amount of bleeding during surgery, between postoperative walking distance and pre- and postoperative vertebral slipping scores.

In patients with one-level LSS, there was a positive correlation between preoperative VAS and preoperative ODI scores, between postoperative VAS and postoperative ODI scores, between postoperative VAS score and pre- and postoperative vertebral slipping scores, between postoperative ODI score and pre- and postoperative slipping scores, between walking distance value and the amount of bleeding during surgery, and between hospitalization time and BMI. There was a negative correlation between preoperative VAS scores and the amount of bleeding during surgery, and between BDUA with discectomy and the amount of bleeding during surgery.
No significant correlation was found among the values in patients with two-level LSS.

\section{DISCUSSION}

In general, the traditional surgery for LSS involves a laminectomy with foraminotomy, and partial facetectomy. In addition, fusion with instrumentation is required when the patient has spondylolisthesis (5). BDUA, which was first described by Spetzger et al. in 1997, is a relatively new minimally invasive technique for the surgical treatment of LSS (20). Nerland et al. compared this minimally invasive approach to the standard laminectomy technique in central lumbar stenosis and reported equivalent effectiveness of both techniques with favourable patient outcomes at one year follow-up (15). This technique incorporates a midline incision, opening the fascia, retracting the muscles, hemilaminectomy, bilateral flavectomy, and decompression $(5,18)$. Since BDUA has several advantages such as less blood loss, a shorter stay in hospital, lower complication rates, and less traumatization of surrounding tissues, it has been increasingly applied by neurosurgeons for the treatment of LSS over the past two decades $(1,4,13,14,16,19,22)$. However, this technique has some disadvantages such as accidental dural tears, root 
damage and cerebrospinal fluid leakage associated with the limited operation field and visualization of critical structures. Therefore, this technically challenging procedure is associated with a steep learning curve and requires considerable experience (16).

In this study, the clinical and demographic features of patients who underwent BDUA are described with the aim of helping in the decision-making for surgery. According to the results of this study, 38 patients $(71.7 \%)$ underwent one-level and 15 patients $(28.3 \%)$ underwent two-level decompression. Microdiscectomy was required in $10(18.2 \%)$ patients without spondylolisthesis. In these patients, no spondylolisthesis was observed in the long-term follow-up. In respect of side-effects or complications, no neurological deficit or dural tear was observed in any patient. Furthermore, neither revision surgery nor fusion (instrumentation) surgery was performed to any patient during the 3-year follow-up period.

LSS is more common in the geriatric population and in many studies the mean age of the sample size is more than 65 years. Recent studies which have compared traditional surgery to BDUA for patients aged 80 years and older have demonstrated that there were no between-group differences in duration and outcome results of the surgical procedures (9). In the current study, unlike previous studies, patients were separated according to age as an elderly group ( $>60$ years) and an adult group ( $<60$ years). Although the elderly cases had advanced stages of degeneration, both groups showed similar improvements. This highlights that BDUA could also be effective in elderly patients. The mean surgery duration was approximately 30 minutes with minimal bleeding (approximately $50 \mathrm{ml}$ per patient), which can be attributed to the patient prone position, and the minimal invasiveness of the surgical approach. Overall, the study results revealed that BDUA was very effective in reducing pain and improving the ODI scores and walking distance in all the patients included in this study. In adult patients, a higher BMI value and/or the application of microdiscectomy could prolong hospitalization. Furthermore, in these patients, preoperative pain could increase the preoperative disability level and the low level preoperative walking distance value could decrease the postoperative walking distance value. It was thought that postoperative VAS scores could be high if the patients had degenerative spondylolisthesis. In the elderly patients, the postoperative disability level was found to be high if preoperative degenerative spondylolisthesis was detected and the postoperative disability level was also related to the postoperative pain level. The postoperative disability level also increased in patients with degenerative spondylolisthesis diagnosed preoperatively. Furthermore, detection of degenerative spondylolisthesis and/or increased preoperative pain level could have a negative effect on walking distance values in these patients.

BDUA without instrumentation has been suggested for selected cases in the presence of spondylolisthesis (5). The transmuscular technique that utilizes tubular retractors causes less destabilization in BDUA $(4,18)$. Recent studies have reported that in patients with LSS and spondylolisthesis, minimally invasive decompression is associated with lower reoperation and fusion rates, less slip progression, and greater patient satisfaction than open surgery $(11,18)$. Likewise, in the current study, hyperflexion-hyperextension dynamic radiographs were taken to assess slipping in all patients preoperatively to check for displacement, rotation, or angulation and BDUA was applied to 19 patients with spondylolisthesis (2). Although the MRI and X-ray results were not compared in this study, it can be said that the MRI findings and X-ray findings did not correlate in some cases. Therefore, the present study findings suggest that BDUA did not cause an increase in slipping. Furthermore, there was no need to perform instrumentation in patients with degenerative spondylolisthesis when the patients were evaluated according to the presence or not of spondylolisthesis from the examination of plain radiographs and clinical findings in the decision of whether or not instrumentation was necessary. Moreover, this technique could lead to improvements in the postoperative VAS score, ODI score and walking distance compared to the preoperative values in these patients. Therefore, it can be said that good results can be obtained without instrumentation if patients with spinal stenosis have no slipping on dynamic $x$-ray, and the spondylolisthesis grade did not change during the 3-year follow-up if patients had degenerative spondylosisthesis. However, the correlation test results suggested that degenerative spondylolisthesis detected preoperatively in male patients could increase the patient disability level and decrease the walking distance values postoperatively compared to the results of patients without spondylolisthesis. In these patients, comorbid diseases such as diabtes mellitus or respiratory diseases could necessitate a longer period of hospitalization. However, degenerative spondylolisthesis detected preoperatively in female patients only seemed to cause an increase in the postoperative pain level. In these patients, preoperative pain could increase the patient's disability. Overall, one-level degenerative spondylolisthesis in particular that was detected preoperatively could negatively affect the postoperative pain level and disability level while decreasing the patient walking distance value.

Finally, this study, which aimed to explore the effects of BDUA in patients with LSS, revealed three main findings:1) BDUA is quite effective in reducing pain, and improving walking distance and disability; 2) there was a significant improvement in the clinical parameters of patients with degenerative spondylolisthesis, with no worsening of the slip; and 3) elderly patients showed similar improvements to adults.

\section{Limitations}

There were some drawbacks to this study. First, walking distance should be measured with a valid and reliable scale rather than from patient statements. Second, although the sample size was large enough compared with previous studies, the number of patients with degenerative spondylolisthesis aged $>65$ years was low. Third, to obtain strong and objective results, there should have been a control group applied with traditional surgery and the follow-up period of the patients could have been longer. Fourth, cost utility assessment or 
cost-effectiveness was not evaluated in the study. However, the study results demonstrated that mean surgery duration was approximately 30 mins, with minimal bleeding and the median duration of hospitalization was 1 to 2 days. That there was no need for instrumentation, a short length of stay in hospital and a low rate of surgical complications supported the idea that this surgical procedure could be cost-efficient. Fifth, the retrospective nature of the study was an inherent weakness. Nevertheless, the results are quite striking and remarkable in terms of explaining the advantages of this surgical method.

\section{CONCLUSION}

In patients with LSS, especially geriatric patients and/ or females, BDUA could result in less intraoperative blood loss and a short stay in hospital and it could be quite effective in reducing pain, improving walking distance and quality of life without producing significant side-effects. Moreover, there was no worsening of the grade or degree of slipping in patients with degenerative spondylolisthesis. Further studies considering BDUA in prospective and randomized-controlled designs are awaited.

\section{- REFERENCES}

1. Alimi M, Hofstetter CP, Torres-Campa JM, Navarro-Ramirez R, Cong GT, Njoku I Jr, Härtl R: Unilateral tubular approach for bilateral laminotomy: Effect on ipsilateral and contralateral buttock and leg pain. Eur Spine J 26: 389-396, 2017

2. Balderston RA, Auerbach JD: The Definition of Lumbar Spinal Instability and Its Clinical Significance. Semin Spine Surg 17: 240-242, 2005

3. Boonstra AM, Schiphorst Preuper HR, Reneman MF, Posthumus JB, Stewart RE: Reliability and validity of the visual analogue scale for disability in patients with chronic musculoskeletal pain. Int J Rehabil Res 31: 165-9, 2008

4. Boukebir MA, Berlin CD, Navarro-Ramirez R, Heiland T, Schöller K, Rawanduzy C, Kirnaz S, Jada A, Härtl R: TenStep Minimally Invasive Spine Lumbar Decompression and Dural Repair Through Tubular Retractors. Oper Neurosurg (Hagerstown) 13: 232-245, 2017

5. Burgstaller JM, Porchet F, Steurer J, Wertli MM: Arguments for the choice of surgical treatments in patients with lumbar spinal stenosis-a systematic appraisal of randomized controlled trials. BMC Musculoskelet Disord 16: 96, 2015

6. Dohzono S, Toyoda H, Matsumura A, Terai H, Suzuki A, Nakamura $\mathrm{H}$ : Clinical and Radiological Outcomes after Microscopic Bilateral Decompression via Unilateral Approach for Degenerative Lumbar Disease: Minimum 5-Year FollowUp. Asian Spine J 11: 285-293, 2017

7. Edward C. Benzel, M. PélegrinEdward C. Benzel: SPINE SURGERY: Tecniques, Complication, Avoidance, and Management". Volume one (Second edition). Elsevier Health Sciences, 2004.

8. Fairbank JC, Pynsent PB: The Oswestry Disability Index. Spine 25: 2940-52, 2000
9. Giannadakis C, Solheim O, Jakola AS, Nordseth T, Gulati AM, Nerland US, Nygaard ØP, Solberg TK, Gulati S: Surgery for Lumbar Spinal Stenosis in Individuals Aged 80 and Older: A Multicenter Observational Study. Am Geriatr Soc 64: 20112018, 2016

10. Hu W, Zhao J, Gong C, Zou M, Yuan JH, Liu XY: A comparison of efficacy between unilateral laminectomy approach bilateral decompression and traditional total laminectomy decompression in the treatment of lumbar spinal stenosis. Zhonghua Yi Xue Za Zhi 96: 1673-6, 2016

11. Kelleher MO, Timlin M, Persaud O, Rampersaud YR: Success and failure of minimally invasive decompression for focal lumbar spinal stenosis in patients with and without deformity. Spine 35: 981-7, 2010

12. Machado GC, Ferreira PH, Yoo RI, Harris IA, Pinheiro MB, Koes BW: Surgical options for lumbar spinal stenosis. Cochrane Database Syst Rev 11: CD012421, 2016

13. Moisi M, Fisahn C, Tkachenko L, Tubbs RS, Ginat D, Grunert P, Jeyamohan S, Reintjes S, Ajayi O, Page J, Oskouian RJ, Hanscom D: Unilateral Laminotomy with Bilateral Spinal Canal Decompression for Lumbar Stenosis: A Technical Note. Cureus 8: 623, 2016

14. Müslüman AM, Cansever T, Yılmaz A, Çavuşoğlu H, Yüce i, Aydın Y: Midterm outcome after a microsurgical unilateral approach for bilateral decompression of lumbar degenerative spondylolisthesis. J Neurosurg Spine 16: 68-76, 2012

15. Nerland US, Jakola AS, Solheim O, Weber C, Rao V, Lønne G, Solberg TK, Salvesen $\varnothing$, Carlsen SM, Nygaard ØP, Gulati S: Minimally invasive decompression versus open laminectomy for central stenosis of the lumbar spine: Pragmatic comparative effectiveness study. BMJ 350: 1603, 2015

16. Phan K, Mobbs RJ: Minimally Invasive Versus Open Laminectomy for Lumbar Stenosis: A Systematic Review and Meta-Analysis. Spine 41: 91-100, 2016

17. Phan K, Teng I, Schultz K, Mobbs RJ: Treatment of Lumbar Spinal Stenosis by Microscopic Unilateral Laminectomy for Bilateral Decompression: A Technical Note. Orthop Surg 9: 241-246, 2017

18. Schöller K, Alimi M, Cong GT, Christos P, Härtl R: Lumbar Spinal Stenosis Associated With Degenerative Lumbar Spondylolisthesis: A Systematic Review and Meta-analysis of Secondary Fusion Rates Following Open vs Minimally Invasive Decompression. Neurosurgery 80: 355-367, 2017

19. Schöller K, Steingrüber T, Stein M, Vogt N, Müller T, PonsKühnemann J, Uhl E: Microsurgical unilateral laminotomy for decompression of lumbar spinal stenosis: longterm results and predictive factors. Acta Neurochir (Wien) 158: 1103-13, 2016

20. Spetzger $U$, Bertalanffy $H$, Naujokat $C$, von Keyserlingk DG, Gilsbach JM: Unilateral laminotomy for bilateral decompression of lumbar spinal stenosis. Part I: Anatomical and surgical considerations. Acta Neurochir (Wien) 139: 3926, 1997

21. Yakut E, Düger T, Oksüz C, Yörükan S, Ureten K, Turan D, Tüzün F, Kiraz S, Krd N, Kayhan H, Yakut Y, Güler Ç: Validation of the Turkish version of the Oswestry Disability Index for patients with low back pain. Spine 29: 581-585, 2004

22. Yaman O, Ozdemir N, Dagli AT, Acar E, Dalbayrak S, Temiz C: A Comparison of Bilateral Decompression via Unilateral Approach and Classic Laminectomy in Patients with Lumbar Spinal Stenosis: A retrospective Clinical Study. Turk Neurosurg 25: 239-45, 2015 